\title{
THERMODYNAMICS AND THE SUPERCRITICAL STEFAN EQUATIONS WITH NUCLEATIONS
}

\author{
BY \\ MORTON E. GURTIN \\ Carnegie Mellon University, Pittsburgh, Pennsylvania
}

1. Introduction. The classical Stefan problem, for two-phase heat conduction in a region $\mathscr{B}$ in $\mathbb{R}^{n}$, is based on balance of energy

$$
\dot{\varepsilon}=-\operatorname{div} \mathbf{q}
$$

away from the phase interface, the constitutive equations

$$
\begin{aligned}
& \varepsilon=\varepsilon_{1}(\theta), \quad \mathbf{q}=-\mathbf{K}_{1}(\theta) \nabla \theta \quad \text { in the solid, } \\
& \varepsilon=\varepsilon_{2}(\theta), \quad \mathbf{q}=-\mathbf{K}_{2}(\theta) \nabla \theta \quad \text { in the liquid, }
\end{aligned}
$$

the local equilibrium hypothesis

$$
[\theta]=0,
$$

and the free-boundary conditions

$$
l V=[q] \cdot \mathbf{m}, \quad \theta=\theta_{0} \quad \text { on the interface, }
$$

with the solid region $\mathscr{B}_{1}(t)$, the liquid region $\mathscr{B}_{2}(t)$, and the interface $\mathscr{S}(t)$ given by

$$
\begin{aligned}
& \mathscr{B}_{1}(t)=\left\{x: \theta(x, t)<\theta_{0}\right\}, \\
& \mathscr{B}_{2}(t)=\left\{x: \theta(x, t)>\theta_{0}\right\}, \\
& \mathscr{S}(t)=\left\{x: \theta(x, t)=\theta_{0}\right\} .
\end{aligned}
$$

Here $\varepsilon(x, t)$ is the internal energy, $\theta(x, t)$ is the temperature, $\mathbf{q}(x, t)$ is the heat flux, $\theta_{0}$ is the transition temperature,

$$
l=\varepsilon_{2}\left(\theta_{0}\right)-\varepsilon_{1}\left(\theta_{0}\right)
$$

is the latent heat; $\mathbf{m}(x, t)$ is the unit normal and $V(x, t)$ the normal velocity for the phase interface with $\mathbf{m}$ directed outward from the solid; and [] denotes the jump across $\mathscr{S}(t)$ (liquid minus solid).

The standard weak formulation of Eqs. (1.1)-(1.6) consists in interpreting the PDE

$$
\tilde{\varepsilon}(\theta)^{\cdot}=-\operatorname{div} \tilde{\mathbf{q}}(\theta, \nabla \theta)
$$

Received March 16, 1992.

1991 Mathematics Subject Classification. Primary 80A22; Secondary 73B30.

(C)1994 Brown University 
in the sense of distributions, with $\tilde{\varepsilon}(\theta)=\varepsilon_{1}(\theta)$ for $\theta \leq \theta_{0}$ and $\tilde{\varepsilon}(\theta)=\varepsilon_{2}(\theta)$ for $\theta>\theta_{0}$, and similarly for $\tilde{\mathbf{q}}(\theta, \nabla \theta)$. In this formulation the solid and liquid regions and the interface are defined, a posteriori, through Eq. (1.5).

It is possible to cool liquids below the transition temperature $\theta_{0}$ and to heat solids above $\theta_{0}$. Supercritical behavior of this type is still described by Eqs. (1.1)-(1.4), but we can no longer define the phase regions and the interface through Eq. (1.5); now the solid (or liquid) region must be separately tracked. Writing

$$
\begin{aligned}
& \chi(x, t)=1, \quad x \in \mathscr{B}_{1}(t), \\
& \chi(x, t)=0, \quad \text { otherwise }
\end{aligned}
$$

for the characteristic function of the solid, and defining

$$
\begin{aligned}
\tilde{\varepsilon}(\theta, \chi) & =\chi \varepsilon_{1}(\theta)+(1-\chi) \varepsilon_{2}(\theta), \\
\tilde{\mathbf{q}}(\theta, \nabla \theta, \chi) & =-\chi \mathbf{K}_{1}(\theta) \nabla \theta-(1-\chi) \mathbf{K}_{2}(\theta) \nabla \theta,
\end{aligned}
$$

we can write the bulk equations (1.1), (1.2) and the interface condition (1.4), as a distributional equation

$$
\tilde{\varepsilon}(\theta, \chi)^{\cdot}=-\operatorname{div} \tilde{\mathbf{q}}(\theta, \nabla \theta, \chi),
$$

but now the interface condition

$$
\theta=\theta_{0} \quad \text { on } \mathscr{S}(t)
$$

must be adjoined to Eq. (1.10). We will refer to Eqs. (1.10) and (1.11) as the supercritical Stefan equations. The free-boundary condition (1.11) is unfortunate, as its locality compromises the usefulness of the distributional equation (1.10).

The object of this paper is to discuss the supercritical Stefan equations within a thermodynamical framework (cf. [11]), the crucial ingredient being an appropriate entropy inequality (the Clausius-Duhem inequality) and, in some cases, an entropy balance. For single-phase heat conduction an entropy balance is a direct consequence of balance of energy and the underlying constitutive equations, but for two-phase materials this inequality is an independent postulate, essentially equivalent to Eq. (1.11) when the interface is dissipationless. In fact, this entropy balance yields a distributional PDE which with Eq. (1.10) provides a weak formulation of the supercritical Stefan equations.

The richness of the supercritical equations emerges when these equations are interpreted within a thermodynamical setting, for then it is possible to extend the development to include phenomena such as nucleations. ${ }^{1}$ Here nucleations are instantaneous, entropy-producing, isoenergetic phase changes over regions of nonzero volume, a definition that includes the instantaneous formation of, say, solid particles

\footnotetext{
${ }^{1}$ Luckhaus [14] was apparently the first to use the fact that nucleations furnish weak solutions of the energy balance, although the basic idea seems due to Visintin [18, 19]. Luckhaus [14] and Almgren (private communication), working within a framework that includes interfacial free-energy, allow for isoenergetic phase changes that (in a certain sense) yield positive entropy production for the body as a whole; unfortunately, their procedure-although a major development in the solution of such problems-does not ensure that the entropy production in arbitrary parts of the body be nonnegative, so that thermodynamically inadmissible solutions are not ruled out.
} 
in sufficiently supercooled liquid as well as situations in which an existing interface jumps.

The entropy inequality is based on a general nonlinear formulation of the basic equations. Most applications, however, are based on an energy equation-linear in each phase-that models behavior near $\theta_{0}$. With this in mind, I formulate the thermodynamics in a manner that is applicable to the general nonlinear theory as well as to the approximate model with linear energy equation. I believe that this formulation, in which a suitable Gibbs function replaces the entropy, is new.

Initial/boundary-value problems associated with the supercritical Stefan equations have been studied by many authors (e.g., Sherman [15]; Friedman [10]; Fasano and Primicerio [7]; Fasano, Primicerio, and Lacey [9]; Howison, Ockendon, and Lacey [12]; Fasano, Primicerio, Howison, and Ockendon [8]). An important result (established in various stages by Sherman [15], Fasano and Primecerio [7], and Fasano, Primicerio, and Lacey [9]) is that liquid temperatures that are sufficiently low can result in unbounded interfacial velocities in finite time. I believe that such unbounded velocities signal a desire of the material to undergo nucleations and that the theory developed here can be used to extend the solution past the "blow-up time".

I not only generalize the supercritical Stefan equations to allow for nucleations, I also develop a theory in which nucleations take place whenever possible. This leads to a set of equations, the nucleated Stefan equations, which, for the simple theory that models behavior near $\theta_{0}$, have the form

$$
\begin{array}{lll}
\dot{u}=\Delta u, & u \leq \frac{1}{2} & \text { in the solid, } \\
\dot{u}=\Delta u, & u \geq-\frac{1}{2} & \text { in the liquid, } \\
V=-[\nabla u] \cdot m, & u=0 & \text { on the interface, }
\end{array}
$$

where $u=\left(\theta-\theta_{0}\right) / \theta_{0}$, while $\Delta$ is the Laplacian.

My final step is to stabilize further the Stefan equations by allowing for fine phase mixtures. This is formally equivalent to replacing the constitutive equations for the entropy with a single convex relation and reduces the resulting free-boundary problem to a standard problem that allows for mushy zones containing fine phase mixtures of liquid and solid.

2. Single-phase thermodynamics. To fix ideas, we consider first a homogeneous single-phase heat conductor that occupies a region $\mathscr{B}$ in $\mathbb{R}^{n}$ and we let $\varepsilon(x, t)$ denote the internal energy, $\theta(x, t)$ the temperature, $q(x, t)$, the heat flux, and $r(x, t)$ the external heat supply.

The first two laws of thermodynamics are balance of energy

$$
\left\{\int_{\mathscr{P}} \varepsilon d V\right\}^{\cdot}=-\int_{\partial \mathscr{P}} \mathbf{q} \cdot \mathbf{n} d A+\int_{\mathscr{P}} r d V
$$

and growth of entropy

$$
\left\{\int_{\mathscr{P}} \eta d V\right\}^{\cdot} \geq-\int_{\partial \mathscr{P}} \theta^{-1} \mathbf{q} \cdot \mathbf{n} d A+\int_{\mathscr{P}} \theta^{-1} r d V
$$


for every part $\mathscr{P}$ (subset of $\mathscr{B}$ ), where $\mathbf{n}$ is the outward unit normal to $\partial \mathscr{P}$. Granted sufficient regularity, these global laws are equivalent to the local laws

$$
\begin{aligned}
& \dot{\varepsilon}=-\operatorname{div} \mathbf{q}+r, \\
& \dot{\eta} \geq-\operatorname{div}\left(\theta^{-1} \mathbf{q}\right)+\theta^{-1} r .
\end{aligned}
$$

For our purposes it is most convenient to consider constitutive equations in which the temperature and the entropy are functions of the internal energy ${ }^{2}$ and the heat flux is given by Fourier's law,

$$
\theta=\hat{\theta}(\varepsilon), \quad \eta=\hat{\eta}(\varepsilon), \quad q=-\mathbf{K}(\theta) \nabla \theta
$$

with $\mathbf{K}(\theta)$, assumed symmetric and invertible, the conductivity tensor. The heat supply $r$ is not prescribed by a constitutive equation but, instead, is arbitrary.

Given a smooth energy field $\varepsilon(x, t)$, the constitutive equations can be used to compute corresponding fields $\theta(x, t), \eta(x, t)$, and $q(x, t)$; balance of energy (2.3) then tells us the heat $r(x, t)$ that must be supplied to support this constitutive process. The second law (2.4) remains to be satisfied; if we assume that all constitutive processes are consistent with Eq. (2.4), then, by substituting the constitutive equations into the inequality

$$
\eta^{\cdot}-\theta^{-1} \dot{\varepsilon}-\theta^{-2} \mathbf{q} \cdot \nabla \theta \geq 0
$$

obtained by eliminating $r$ between Eqs. (2.3) and (2.4), we are led to the temperatureentropy relation

$$
\hat{\theta}(\varepsilon)=\hat{\eta}^{\prime}(\varepsilon)^{-1}
$$

in conjunction with the standard restriction

$$
\mathbf{K}(\theta) \text { positive definite. }
$$

The restrictions (2.7) and (2.8) are necessary and sufficient that smooth "constitutive processes" consistent with balance of energy obey the second law. (Cf. Coleman and Noll [3]. Note that Eq. (2.7) is the classical relation $d \varepsilon=\theta d \eta$.) Thus, granted Eqs. (2.7) and (2.8), we can omit all mention of the second law and simply consider balance of energy. This will not generally be true when we study two-phase materials.

Note that, because of the constitutive restrictions (2.7) and (2.8), and by the energy balance (2.3), the entropy inequality (2.4) reduces to an entropy balance

$$
\begin{aligned}
\dot{\eta} & =-\operatorname{div}\left(\theta^{-1} \mathbf{q}\right)+\theta^{-1} r+\gamma, \\
\gamma & =\theta^{-2} \nabla \theta \cdot \mathbf{K}(\theta) \nabla \theta \geq 0,
\end{aligned}
$$

with $\gamma$ the local entropy production. We emphasize that, in contrast to Eq. (2.4), which is basic, Eqs. (2.9) are a consequence of the underlying constitutive theory.

The requirement that the temperature be strictly positive yields the restriction $\hat{\eta}^{\prime}>0$; we assume, in addition, that

$$
\hat{\eta}^{\prime \prime}<0
$$

\footnotetext{
${ }^{2}$ The use of internal energy as an independent variable (rather than temperature) facilitates our study of nucleations, which for us are instantaneous isoenergetic phase changes over regions of nonzero volume.
} 
which ensures that-as functions of energy - the entropy is strictly concave, the temperature is strictly increasing. The relation $\theta=\hat{\theta}(\varepsilon)$ then has an inverse $\varepsilon=\hat{\varepsilon}(\theta)$; writing

$$
C(\theta)=\hat{\varepsilon}^{\prime}(\theta)>0
$$

for the specific heat, balance of energy is then equivalent to the classical parabolic PDE

$$
C(\theta) \theta^{\cdot}=\operatorname{div}(\mathbf{K}(\theta) \nabla \theta)+r .
$$

3. Formulation of the single-phase theory in terms of a Gibbs function. Most studies of two-phase heat conduction concern behavior near the transition temperature $\theta_{0}$. Dynamics with small temperature changes is cumbersome using a formulation based on entropy; a more useful version of the second law involves the Gibbs function

$$
\varphi=\varepsilon-\theta_{0} \eta
$$

3.1. Theory for small temperature-deviations. We are chiefly interested in situations involving small departures from a given fixed, constant temperature $\theta_{0}$. With this in mind, we introduce the temperature deviation $u$ defined by the relation

$$
\theta=\theta_{0}(1+u)
$$

Our goal is a theory-appropriate to small temperature deviations-that is consistent with its own version of the second law. To deduce a suitable "growth inequality" for the theory we use Eq. (3.2) with $u$ small to justify replacing $\theta^{-1}$ in the entropygrowth inequality $(2.2)$ by $(1-u) / \theta_{0}$; multiplying the resulting relation by $\theta_{0}$ and using Eqs. (3.1), (3.2), and balance of energy (2.1), we are led to the inequality

$$
\left\{\int_{\mathscr{P}} \varphi d V\right\}^{\cdot} \leq-\int_{\partial \mathscr{P}} u \mathbf{q} \cdot \mathbf{n} d A+\int_{\mathscr{D}} u r d V
$$

for every $\mathscr{P}$. We will refer to Eq. (3.3) as the dissipation inequality; this inequality will play a role analogous to that played by the entropy inequality in the general theory. We will sometimes use Eq. (3.3) in the slightly weaker form

$$
\int_{\mathscr{P}} \varphi\left(\cdot, t_{2}\right) d V-\int_{\mathscr{P}} \varphi\left(\cdot, t_{1}\right) d V \leq-\int_{t_{1}}^{t_{2}} \int_{\partial \mathscr{P}} u \mathbf{q} \cdot \mathbf{n} d A d t+\int_{t_{1}}^{t_{2}} \int_{\mathscr{P}} u r d V d t
$$

for all parts $\mathscr{P}$ and times $t_{2}>t_{1}$, or in the local form

$$
\dot{\varphi} \leq-\operatorname{div}(u \mathbf{q})+u r .
$$

The theory of small temperature deviations is based on balance of energy (2.1) and the dissipation inequality (3.3). In this theory $\varphi$ and $u$ play the roles previously played by $\eta$ and $\theta$; we therefore begin with constitutive equations

$$
u=\hat{u}(\varepsilon), \quad \varphi=\hat{\varphi}(\varepsilon), \quad \mathbf{q}=-\mathbf{M} \nabla u,
$$

with $\mathbf{M} \neq 0$, assumed constant, symmetric, and invertible.

As in the general theory described in Sec. 2.1, we require compatibility of the constitutive equations with thermodynamics; here the requirement is that all smooth 
processes consistent with Eqs. (3.6) and balance of energy (2.3) obey the dissipation inequality (3.5). The results are the restrictions

$$
\hat{u}(\varepsilon)=\hat{\varphi}^{\prime}(\varepsilon), \quad \text { M positive definite. }
$$

A further requirement is that the underlying PDE-obtained by substituting Eqs. (3.6) into balance of energy (2.3) - be linear. We therefore want an affine relation between $u$ and $\varepsilon$, and hence a quadratic relation between $\varphi$ and $\varepsilon$. We assume that there is a unique $\varepsilon_{0}$ with $\hat{u}\left(\varepsilon_{0}\right)=0$, and we define $\varphi=\hat{\varphi}(\varepsilon)$ through Eq. (3.1) with $\eta=\hat{\eta}(\varepsilon)$ approximated to quadratic terms near $\varepsilon=\varepsilon_{0}$; we then define $u=\hat{u}(\varepsilon)$ through Eq. (3.7); the results are

$$
\varphi=\hat{\varphi}(\varepsilon)=\varphi_{0}+\frac{1}{2} B\left(\varepsilon-\varepsilon_{0}\right)^{2}, \quad u=\hat{u}(\varepsilon)=B\left(\varepsilon-\varepsilon_{0}\right),
$$

with

$$
\varphi_{0}=\varepsilon_{0}-\theta_{0} \hat{\eta}\left(\varepsilon_{0}\right), \quad B=-\theta_{0} \hat{\eta}^{\prime \prime}\left(\varepsilon_{0}\right)>0 .
$$

These equations simplify when $u$ is used as an independent variable:

$$
\varphi=\varphi_{0}+\frac{1}{2} c u^{2}, \quad \varepsilon=\varepsilon_{0}+c u, \quad c=B^{-1} ;
$$

and Eqs. (3.10) with Eqs. (2.3) and (3.6) ${ }_{3}$ yield the classical linear heat equation

$$
c u^{\cdot}=\operatorname{div}(\mathbf{M} \nabla u)+r .
$$

Finally, solutions of Eq. (3.11) are consistent with the dissipation balance

$$
\dot{\varphi}=-\operatorname{div}(u \mathbf{q})+u r-\zeta, \quad \zeta=\nabla u \cdot \mathbf{M} \nabla u \geq 0,
$$

which has the global form

$$
\left\{\int_{\mathscr{P}} \varphi d V\right\}^{\cdot}=-\int_{\partial \mathscr{P}} u \mathbf{q} \cdot \mathbf{n} d A+\int_{\mathscr{P}} u r d V-\int_{\mathscr{P}} \zeta d V
$$

for every part $\mathscr{P}$; the relation (3.12) is an analog of the entropy balance (2.9).

REMARK 3.1. This development is consistent with a direct linearization of Eq. (2.12) near $\theta_{0}$, or, equivalently, a linearization of $\varepsilon=\hat{\varepsilon}(\theta)$ and $\mathbf{q}=-\mathbf{K}(\theta) \nabla \theta$ near $\theta=\theta_{0}$. By Eqs. (2.7), (2.11), (3.9) 2 , and $(3.10)_{3}, c=\theta_{0} C\left(\theta_{0}\right)$, so that the linear approximation of $\theta=\hat{\theta}(\varepsilon)$ near $\varepsilon_{0}$ is $\theta=\theta_{0}+\theta_{0} B\left(\varepsilon-\varepsilon_{0}\right)$; by Eq. (3.1), this is Eq. (3.8) $)_{2}$. The final step in establishing consistency is to identify Eq. (3.6) $)_{3}$ as the linearization of Eq. $(2.5)_{3}$, which yields $\mathbf{M}=\theta_{0} \mathbf{K}\left(\theta_{0}\right)$.

3.2. General theory in terms of a Gibbs function. Although the dissipation inequality (3.3) was derived as an approximation based on the assumption that $u$ be small, this relation follows as an exact consequence of balance of energy (2.1) and growth of entropy (2.2) provided $u$ is defined by ${ }^{3}$

$$
u=\left(\theta-\theta_{0}\right) / \theta
$$

rather than by Eq. (3.2).

\footnotetext{
${ }^{3}$ The relations (3.2) and (3.14) are consistent: writing $u$ for Eq. (3.14) and $u_{\text {small }}$ for Eq. (3.2), we see that $u=u_{\text {small }}+O\left(u_{\text {small }}^{2}\right)$.
} 
Granted Eqs. (3.1) and (3.14), the general theory can equivalently be formulated in terms of the Gibbs function $\varphi$ and the temperature deviation $u$ using balance of energy (2.1) and the dissipation inequality (3.3) as basic laws. The constitutive theory begins with Eqs. (3.6), but with $\mathbf{M}=\mathbf{M}(u)$, and reduces, as in the paragraph following Eqs. (3.6), to

$$
\hat{u}(\varepsilon)=\hat{\varphi}^{\prime}(\varepsilon), \quad \mathbf{q}=-\mathbf{M}(u) \nabla u, \quad \mathbf{M}(u) \text { positive definite. }
$$

These results are equivalent to Eqs. (2.5), (2.7), and (2.8). By Eq. (3.1), the analog of the assumption $(2.10)$ is

$$
\hat{\varphi}^{\prime \prime}>0 \text {, }
$$

which implies that

$$
u=\hat{u}(\varepsilon) \text { has an inverse } \varepsilon=\hat{\varepsilon}(u) ;
$$

as before, we write $\varepsilon_{0}=\hat{\varepsilon}(0)$.

Let $\psi=\hat{\psi}(\varepsilon)$ denote the constitutive equation for the free energy

$$
\psi=\varepsilon-\theta \eta \text {. }
$$

Then, as a consequence of Eqs. (3.1), (3.14), (3.15) , and (3.16), we have the following:

Properties of the Gibbs functions. The Gibbs function $\hat{\varphi}(\varepsilon)$ is strictly convex with a minimum at $\varepsilon_{0}$, where $\varepsilon_{0}$ is the internal energy corresponding to $u=0 \quad\left(\theta=\theta_{0}\right)$. The minimum value of $\hat{\varphi}(\varepsilon)$ is the free energy at $\varepsilon_{0}$ :

$$
\hat{\varphi}\left(\varepsilon_{0}\right)=\hat{\psi}\left(\varepsilon_{0}\right) \text {. }
$$

We let

$$
c(u)=\hat{\varepsilon}^{\prime}(u)
$$

balance of energy is then equivalent to the PDE

$$
c(u) u^{\dot{ }}=\operatorname{div}(\mathbf{M}(u) \nabla u)+r,
$$

and solutions of Eq. (3.21) automatically satisfy the dissipation balances (3.12) and (3.13).

Finally, the identities

$$
c(u)=\left(\theta^{2} / \theta_{0}\right) C(\theta), \quad \mathbf{M}(u)=\left(\theta^{2} / \theta_{0}\right) \mathbf{K}(\theta)
$$

relate the specific heats and conductivities of the general formulations.

REMARK 3.2. Note that, although the theory of Sec. 3.1 is based on an approximation of small temperature deviations $u$, the resulting Gibbs function $\hat{\varphi}(\varepsilon)$ as defined by Eqs. (3.8) and (3.9) has all of the properties stated above. Most of our discussion will be within the general framework of this section, but all of our results will apply also to the simplified theory based on Eq. (3.8).

REMARK 3.3. A theory based on balance of energy (2.1) and the dissipation inequality (3.3) together with the constitutive equations (3.15) is isomorphic to the continuum theory of (isothermal) mass diffusion with $\varepsilon$ the concentration, $\mathbf{q}$ the mass flux, $r$ the mass supply, $\varphi$ the free energy, and $u$ the chemical potential; Eq. (2.1) is then balance of mass, while Eq. (3.3) is the free-energy inequality appropriate to this theory. 
4. Two-phase constitutive theory.

4.1. General theory based on entropy. We assume that the solid and liquid phases, labelled 1 and 2 , are governed by constitutive equations

$$
\eta=\hat{\eta}_{1}(\varepsilon), \quad \theta=\hat{\theta}_{1}(\varepsilon)=\hat{\eta}_{1}^{\prime}(\varepsilon)^{-1}, \quad \mathbf{q}=-\mathbf{K}_{1}(\theta) \nabla \theta
$$

for the solid and

$$
\eta=\hat{\eta}_{2}(\varepsilon), \quad \theta=\hat{\theta}_{2}(\varepsilon)=\hat{\eta}_{2}^{\prime}(\varepsilon)^{-1}, \quad \mathbf{q}=-\mathbf{K}_{2}(\theta) \nabla \theta
$$

for the liquid, with each set of equations consistent with the assumptions of Sec. 2.1.

We assume that the graphs of $\hat{\eta}_{1}(\varepsilon)$ and $\hat{\eta}_{2}(\varepsilon)$ are as shown in Fig. 1. In particular, we assume that these graphs cross at a single energy $\varepsilon^{*}$; then, since each of the graphs is strictly concave, the composite diagram is convexified by a single line $\mathscr{L}$, with $\mathscr{L}$ tangent to $\hat{\eta}_{1}(\varepsilon)$ at a single point $\varepsilon=\varepsilon_{1}$ and to $\hat{\eta}_{2}(\varepsilon)$ at a single point $\varepsilon=\varepsilon_{2}$. The common temperature

$$
\theta_{0}=\hat{\theta}_{1}\left(\varepsilon_{1}\right)=\hat{\theta}_{2}\left(\varepsilon_{2}\right)
$$

is then the transition temperature, while

$$
l:=\varepsilon_{2}-\varepsilon_{1}
$$

is the latent heat.

The free energy (3.18) is given, in the solid and in the liquid, by

$$
\begin{aligned}
& \hat{\psi}_{1}(\varepsilon)=\varepsilon-\hat{\theta}_{1}(\varepsilon) \hat{\eta}_{1}(\varepsilon), \\
& \hat{\psi}_{2}(\varepsilon)=\varepsilon-\hat{\theta}_{2}(\varepsilon) \hat{\eta}_{2}(\varepsilon),
\end{aligned}
$$

and it follows from the definitions of $\theta_{0}, \varepsilon_{1}$, and $\varepsilon_{2}$ that

$$
\hat{\psi}_{1}\left(\varepsilon_{1}\right)=\varepsilon_{1}-\theta_{0} \hat{\eta}_{1}\left(\varepsilon_{1}\right)=\hat{\psi}_{2}\left(\varepsilon_{2}\right)=\varepsilon_{2}-\theta_{0} \hat{\eta}_{2}\left(\varepsilon_{2}\right),
$$

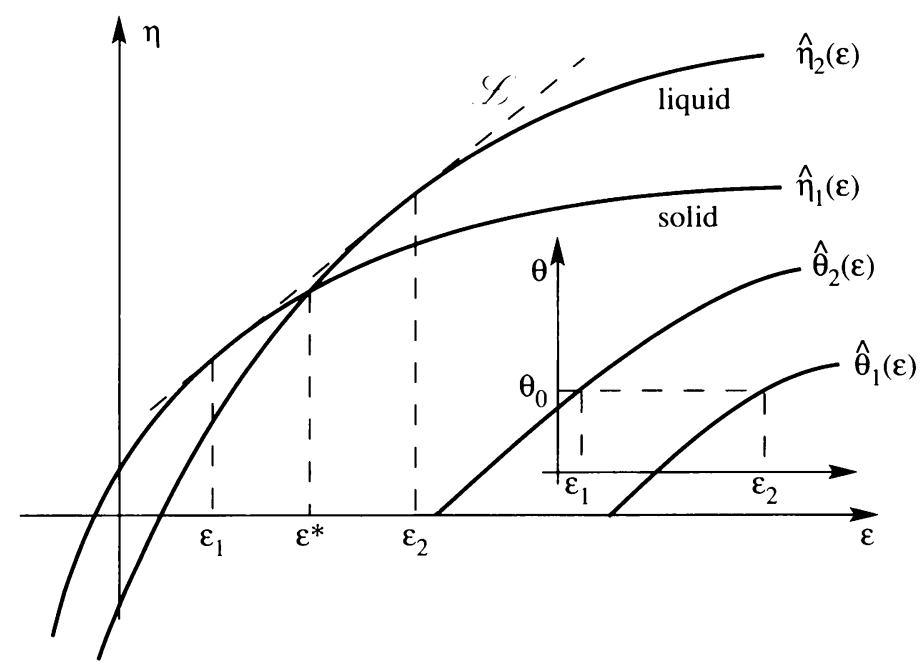

Fig. 1. Constitutive relations for the entropy $\eta$ and temperature $\theta$ as functions of the internal energy $\varepsilon$. 
while the converse assertion

$$
\hat{\theta}_{1}(w)=\hat{\theta}_{2}(z) \quad \& \quad \hat{\psi}_{1}(w)=\hat{\psi}_{2}(z) \quad \Rightarrow \quad w=\varepsilon_{1}, z=\varepsilon_{2}
$$

is a consequence of the strict concavity of $\hat{\eta}_{1}(\varepsilon)$ and $\hat{\eta}_{2}(\varepsilon)$; thus the temperature and free energies of the phases coincide at and only at the transition temperature.

Liquid at energies $\varepsilon<\varepsilon_{2}$ is supercooled as its temperatures lie below the transition temperature; similarly, solid at energies $\varepsilon>\varepsilon_{1}$ is superheated. We will refer to liquid at energies $\varepsilon<\varepsilon^{*}$ as super-supercooled, and to solid at energies $\varepsilon>\varepsilon^{*}$ as supersuperheated. Super-supercooled liquid has $\hat{\eta}_{2}(\varepsilon)<\hat{\eta}_{1}(\varepsilon)$, while super-superheated solid has $\hat{\eta}_{1}(\varepsilon)<\hat{\eta}_{2}(\varepsilon)$; in either case a change of phase at constant energy raises the entropy, making super-supercritical material unstable to isoenergetic perturbations.

4.2. General theory based on the Gibbs function. We now consider the alternative formulation in terms of the Gibbs function $\varphi$ and the temperature deviation ${ }^{4} u$, with $\theta_{0}$ the transition temperature, so that $u=0$ is the temperature-deviation at transition. This formulation is based on the constitutive equations

$$
\varphi=\hat{\varphi}_{1}(\varepsilon), \quad u=\hat{u}_{1}(\varepsilon)=\hat{\varphi}_{1}^{\prime}(\varepsilon), \quad \mathbf{q}=-\mathbf{M}_{1}(u) \nabla u
$$

for the solid and

$$
\varphi=\hat{\varphi}_{2}(\varepsilon), \quad u=\hat{u}_{2}(\varepsilon)=\hat{\varphi}_{2}^{\prime}(\varepsilon), \quad \mathbf{q}=-\mathbf{M}_{2}(u) \nabla u
$$

for the liquid, with each set of equations consistent with the assumptions of Sec. 3.2.

We assume that $\hat{\varphi}_{1}(\varepsilon)$ and $\hat{\varphi}_{2}(\varepsilon)$ are consistent with the assumptions laid down for the entropies in the paragraph following Eqs. (4.2). Then $\varepsilon_{1}$ and $\varepsilon_{2}$ are given by

$$
\hat{u}_{1}\left(\varepsilon_{1}\right)=\hat{u}_{2}\left(\varepsilon_{2}\right)=0 \text {, }
$$

and we may conclude from Eq. (3.1), the properties of the Gibbs function established in Sec. 3.2, and (4.6) that (see Fig. 2 on p. 142): $\hat{\varphi}_{1}(\varepsilon)$ and $\hat{\varphi}_{2}(\varepsilon)$ are strictly convex and cross at, and only at, $\varepsilon^{*} ; \varepsilon=\varepsilon_{1}$ and $\varepsilon=\varepsilon_{2}$ minimize $\hat{\varphi}_{1}(\varepsilon)$ and $\hat{\varphi}_{2}(\varepsilon)$, respectively; and the minimum values of these two functions coincide, so, without loss in generality, we may suppose that

$$
\hat{\varphi}_{1}\left(\varepsilon_{1}\right)=\hat{\varphi}_{2}\left(\varepsilon_{2}\right)=0
$$

super-supercooled liquid has $\hat{\varphi}_{2}(\varepsilon)>\hat{\varphi}_{1}(\varepsilon)$, while super-superheated solid has $\hat{\varphi}_{1}(\varepsilon)$ $>\hat{\varphi}_{2}(\varepsilon)$.

Finally we note that the analog of Eqs. (4.6) and (4.7) within the present formulation is

$$
\hat{u}_{1}(w)=\hat{u}_{2}(z)=U \quad \& \quad \hat{\varphi}_{1}(w)-U w=\hat{\varphi}_{2}(z)-U z \quad \Leftrightarrow \quad w=\varepsilon_{1}, z=\varepsilon_{2} .
$$

\footnotetext{
${ }^{4}$ Defined by Eq. (3.2) in the theory for small temperature deviations and by Eq. (3.14) in the general theory.
} 

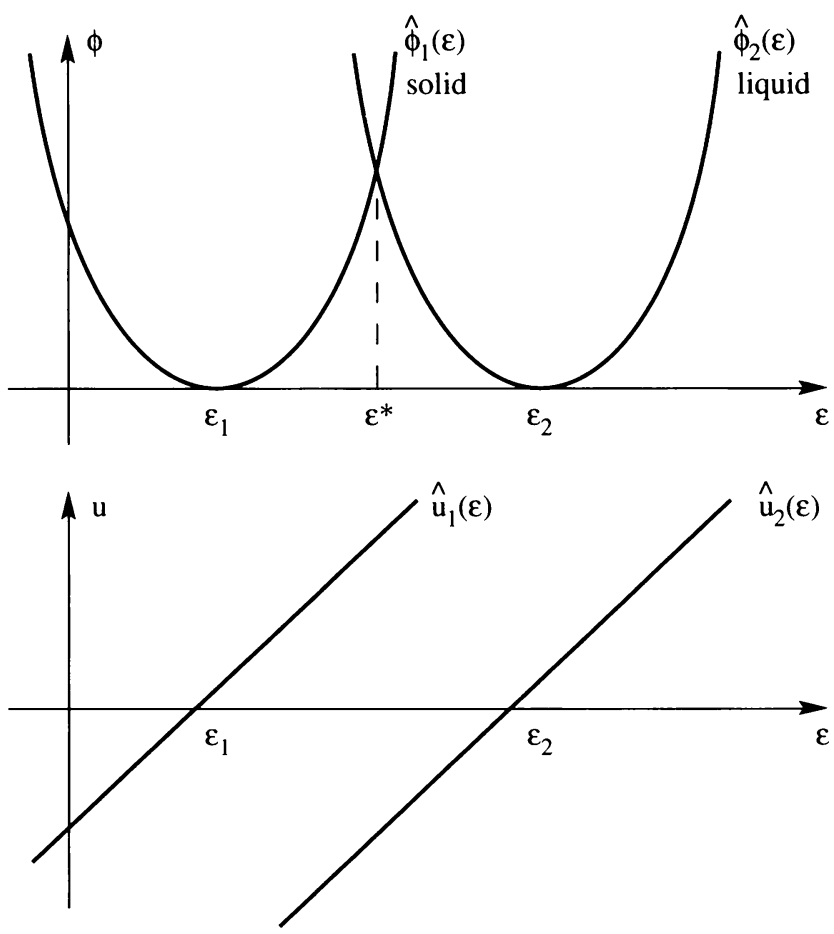

Fig. 2. Constitutive relations for the Gibbs function $\varphi$ and temperature deviation $u$ as functions of the internal energy $\varepsilon$.

4.3. Theory near the transition temperature. Model equations. To model behavior near the transition temperature we use the constitutive equations (3.8), with $\varphi_{0}=0$, in each phase:

$$
\begin{gathered}
\varphi=\hat{\varphi}_{1}(\varepsilon)=\frac{1}{2} B_{1}\left(\varepsilon-\varepsilon_{1}\right)^{2}, \quad u=\hat{u}_{1}(\varepsilon)=B_{1}\left(\varepsilon-\varepsilon_{1}\right), \\
\mathbf{q}=-\mathbf{M}_{1} \nabla u
\end{gathered}
$$

in the solid, and

$$
\begin{gathered}
\varphi=\hat{\varphi}_{2}(\varepsilon)=\frac{1}{2} B_{2}\left(\varepsilon-\varepsilon_{2}\right)^{2}, \quad u=\hat{u}_{2}(\varepsilon)=B_{2}\left(\varepsilon-\varepsilon_{2}\right), \\
\mathbf{q}=-\mathbf{M}_{2} \nabla u
\end{gathered}
$$

in the liquid, or equivalently, letting $c_{i}=B_{i}^{-1}$,

$$
\begin{array}{llll}
\varphi=\frac{1}{2} c_{1} u^{2}, & \varepsilon=\varepsilon_{1}+c_{1} u, & \mathbf{q}=-\mathbf{M}_{1} \nabla u & \text { in the solid, } \\
\varphi=\frac{1}{2} c_{2} u^{2}, & \varepsilon=\varepsilon_{2}+c_{2} u, & \mathbf{q}=-\mathbf{M}_{2} \nabla u & \text { in the liquid. }
\end{array}
$$

Remark 4.1. For $B_{1} \neq B_{2}$ the graphs of $\hat{\varphi}_{1}(\varepsilon)$ and $\hat{\varphi}_{2}(\varepsilon)$ cross not only at the energy $\varepsilon=\varepsilon^{*}$ between $\varepsilon_{1}$ and $\varepsilon_{2}$ but also at a second energy $\varepsilon=\varepsilon^{* *}$. Thus for $B_{1} \neq B_{2}$ this special theory does not fall within the general framework of Sec. 4.2. The crossing at $\varepsilon^{* *}$ is spurious; it has no counterpart within the general theory and introduces an erroneous interchange in the stability of the two phases. In fact, this second crossing induces a spurious phase change, since the convexification of the diagram of $\varphi$ versus $\varepsilon$ has a nonconvex portion around $\varepsilon=\varepsilon^{* *}$. Thus extreme care 
should be taken if $B_{1} \neq B_{2}$ 一solutions that have $u$ in the nonconvex portion around $\varepsilon=\varepsilon^{* *}$ could be both qualitatively and quantitatively incorrect.

In view of this remark, when considering the theory of this section we will generally assume that $B_{1}=B_{2} \quad\left(c_{1}=c_{2}\right)$, which is the physical requirement that the specific heats of the solid and liquid phases coincide at the transition temperature. In fact, we will assume that a scaling has been chosen such that

$$
B_{1}=B_{2}=1 \quad\left(c_{1}=c_{2}=1\right), \quad \varepsilon_{1}=0, \quad \varepsilon_{2}=1 ;
$$

then

$$
l=1, \quad \varepsilon^{*}=\frac{1}{2} .
$$

Further, to have a simple model with a minimum of qualitatively unimportant constants, we will assume, in addition, that the material is isotropic with equal conductivities in the two phases, so that, modulo a further scaling, the constitutive equation for the heat flux is given by

$$
\mathbf{q}=-\nabla u
$$

in each phase, which renders the dissipation $(3.12)_{2}$ of the form

$$
\zeta=|\nabla u|^{2} \text {. }
$$

5. Balance of energy and the dissipation inequality. Weak formulation for nondissipative interfaces.

5.1. Basic equations. The time $t$ will always be confined to a fixed interval $[0, T)$. Let $\mathscr{B}$ consist of a solid region $\mathscr{B}_{1}(t)$ and a liquid region $\mathscr{B}_{2}(t)$ separated by a phase interface $\mathscr{S}(t)$, and let $\mathbf{m}(x, t)$ denote the unit normal and $V(x, t)$ the corresponding normal velocity for $\mathscr{S}(t)$ with $\mathbf{m}$ directed outward from the solid. Further, let $\chi(x, t)$ denote the characteristic function of $\mathscr{B}_{1}(t)$ (cf. Eq. (1.8)), and let $\varepsilon(x, t)$ be an energy consistent with $\chi(x, t)$ in the sense that $\varepsilon(x, t)$ is smooth where $\chi(x, t)$ is smooth and otherwise suffers at most jump discontinuities. Further, let $\varphi(x, t), u(x, t)$, and $\mathbf{q}(x, t)$ be defined through the constitutive equations (4.8) and (4.9), which we now write in the form

$$
\begin{aligned}
& \varphi=\chi \hat{\varphi}_{1}(\varepsilon)+(1-\chi) \hat{\varphi}_{2}(\varepsilon), \\
& u=\chi \hat{u}_{1}(\varepsilon)+(1-\chi) \hat{u}_{2}(\varepsilon), \\
& \mathbf{q}=-\chi \mathbf{M}_{1}(u) \nabla u-(1-\chi) \mathbf{M}_{2}(u) \nabla u .
\end{aligned}
$$

Finally, we assume that, for almost every $t$,

$$
u(x, t) \text { is continuous in } x \text { on } \mathscr{B} \text {. }
$$

This assumption, which is basic to all that follows, will be referred to as the local equilibrium hypothesis.

We neglect the energy and entropy of the interface and therefore consider balance of energy and the dissipation inequality in the forms (2.1) and (3.3). Trivially, the results of Sec. 3.2 hold away from the interface. Balance of energy (2.1) and the constitutive equations therefore yield Eq. (3.3) in each phase, but (without further restrictions) Eq. (3.3) will generally not hold automatically when $\mathscr{P}$ contains the 
interface. $^{5}$ Further, it is not enough simply to require consistency with the second law: constitutive assumptions, which delineate the type of phase interaction under consideration, are needed. Here we will specify such assumptions as restrictions on the manner in which the interface dissipates energy.

Consider first a smoothly evolving interface $\mathscr{S}(t)$. Then Eqs. (2.1), (3.3), and (5.2) yield the relations

$$
\begin{aligned}
\dot{\varepsilon} & =-\operatorname{div} \mathbf{q}+r, \\
\dot{\varphi} & \leq-\operatorname{div}(u \mathbf{q})+u r
\end{aligned}
$$

in bulk (in $\mathscr{B}_{1}(t)$ and in $\mathscr{B}_{2}(t)$ for all $\left.t\right)$ in conjunction with the interface conditions

$$
\begin{gathered}
{[\varepsilon] V=[\mathbf{q}] \cdot \mathbf{m},} \\
{[\varphi] V \geq u[\mathbf{q}] \cdot \mathbf{m}}
\end{gathered}
$$

to be satisfied on $\mathscr{S}(t)$ for all $t$. Here [] denotes the jump across $\mathscr{S}(t)$ (the limit from $\mathscr{B}_{2}(t)$ minus that from $\left.\mathscr{B}_{1}(t)\right)$.

We will consider materials for which smooth interfacial motions of this type are nondissipative. To state this assumption precisely, we define the dissipation $\Gamma(\mathscr{P})$ in any part $\mathscr{P}$ by

$$
\Gamma(\mathscr{P})=\left\{\int_{\mathscr{P}} \varphi d V\right\}^{\cdot}+\int_{\partial \mathscr{P}} u \mathbf{q} \cdot \mathbf{n} d A-\int_{\mathscr{P}} u r d V \leq 0 .
$$

Let $\left\{\mathscr{P}_{n}\right\}$ be a sequence of parts none of which contain the interface at time $t$. Then, by Eq. (3.13), which holds in the individual phases,

$$
\Gamma\left(\mathscr{P}_{n}\right)(t) \rightarrow 0 \text { if volume }\left(\mathscr{P}_{n}\right) \rightarrow 0 \text {. }
$$

Our hypothesis of a nondissipative interface is the requirement that (5.6) be satisfied for all sequences $\left\{\mathscr{P}_{n}\right\}$, irrespective of whether or not the $\mathscr{P}_{n}$ 's contain the interface. $^{6}$

This assumption has strong consequences. It implies a stronger version of Eq. $(5.4)_{2}$, namely,

$$
[\varphi] V=u[q] \cdot \mathbf{m} ;
$$

and, since Eq. (3.13) holds in $\mathscr{B}_{1}(t)$ and $\mathscr{B}_{2}(t)$, we may use Eq. (5.7) to show that the dissipation balance (3.13) holds for all parts $\mathscr{P}$; in fact,

$$
\Gamma(\mathscr{P})=-\int_{\mathscr{P}} \zeta d V
$$

Further, Eqs. (5.4) 1 and (5.7) yield

$$
\{[\varphi]-u[\varepsilon]\} V=0,
$$

\footnotetext{
${ }^{5}$ An analogous situation occurs in the theory of shock waves: growth of entropy holds automatically away from a shock but must be adopted as an independent hypothesis across such waves.

${ }^{6}$ There are theories of interfacial behavior in which the interface is dissipative; e.g., interfacial kinetics is often modelled by allowing the limit (5.7) to have the form $b(\theta, V) V^{2}(b>0)$ when $\mathscr{P}$ approaches the interface (cf., e.g., Gurtin [11]).
} 
and, appealing to Eqs. (4.10), (4.12), and (5.2), we see that, for $V \neq 0$, the temperature deviation obeys the classical Stefan condition $u=0$. However, we do not have the classical Stefan problem, since the temperature deviation is not required to be $\geq 0$ in the liquid and $\leq 0$ in the solid. What we have shown is that, [11, Remark 6.1] granted balance of energy and the local equilibrium condition (5.2),

the Stefan condition $u=0$ is a consequence

of the balance law for entropy.

Further, Eqs. (4.4) and (4.10) and the Stefan condition yield $[\varepsilon]=l$, so that Eq. (5.4), becomes $l V=[q] \cdot \mathbf{m}$.

Summarizing, we have the system

$$
\begin{aligned}
\dot{\varepsilon} & =-\operatorname{div} \mathbf{q}+r & & \text { in bulk, } \\
l V & =[\mathbf{q}] \cdot \mathbf{m}, \quad u=0 & & \text { on the interface }
\end{aligned}
$$

supplemented by Eqs. (5.1) and (5.2). Conversely, solutions of this system satisfy the energy and dissipation balances (2.1) and (3.3).

Thus the global balances furnish a weak form of the system (5.10). A possibly more useful formulation utilizes the distributional form of these balances. If $w, \mathbf{h}$, and $p$ are smooth away from the interface and have at most jump discontinuities across the interface, then $w^{\circ}=-\operatorname{div} \mathbf{h}+p$ in the sense of distributions on $\mathscr{B} \times$ $[0, T)$ if and only if this equation is satisfied classically away from the interface and $[w] V=[h] \cdot \mathbf{m}$ across the interface (cf. Dafermos [5] for a discussion of distributional balance and growth laws and their relation to integral laws such as Eqs. (2.1) and (3.3) and to jump conditions such as Eq. (5.4)). Using this result, we are led to the following proposition, in which $\varepsilon(x, t)$ is consistent with $\chi(x, t)$, in which the local equilibrium hypothesis (5.2), the constitutive equations (5.1), and the constitutive assumptions stated in Sec. 4.2 are tacit, in which $\zeta$ is given by Eqs. (3.12), and in which the interface $\mathscr{S}(t)$ evolves smoothly.

Alternative formulations of the supercritical Stefan equations. The following are equivalent:

(i) $(\varepsilon, \chi)$ satisfies balance of energy (2.1) and the dissipation inequality (3.3), and the interface is nondissipative in the sense of (5.6);

(ii) $(\varepsilon, \chi)$ satisfies the energy and dissipation balances (2.1) and (3.13);

(iii) $(\varepsilon, \chi)$ satisfies the Stefan system $(5.10)$;

(iv) $(\varepsilon, \chi)$ satisfies the balance laws

$$
\begin{aligned}
\dot{\varepsilon} & =-\operatorname{div} \mathbf{q}+r, \\
\dot{\varphi} & =-\operatorname{div}(u \mathbf{q})+u r-\zeta
\end{aligned}
$$

in the sense of distributions.

The distributional equations (5.11) make sense under regularity conditions far less stringent than those used in their derivation, and they apply in the presence of multiple interfaces that may merge or disappear. We will refer to Eqs. (5.11) supplemented by the constitutive equations and by (5.2) (for almost all $t$ ) as the supercritical Stefan equations. 
5.2. Model equations for behavior near the transition temperature. In terms of the simple theory based on the model equations (4.13)-(4.18), the constitutive relations (5.1) have the form

$$
\begin{aligned}
2 \varphi & =\chi \varepsilon^{2}+(1-\chi)(\varepsilon-1)^{2}, \\
u & =\chi \varepsilon+(1-\chi)(\varepsilon-1), \\
\mathbf{q} & =-\nabla u
\end{aligned}
$$

or, equivalently,

$$
\begin{aligned}
2 \varphi & =u^{2}, \\
\varepsilon & =\chi u+(1-\chi)(1+u), \\
\mathbf{q} & =-\nabla u .
\end{aligned}
$$

In this case Eqs. (5.10) reduce to

$$
\begin{aligned}
& \dot{u}=\Delta u+r \quad \text { in bulk, } \\
& V=-[\nabla u] \cdot \mathbf{m}, \quad u=0 \quad \text { on the interface, }
\end{aligned}
$$

while the distributional equations (5.11) take the form

$$
\begin{aligned}
\dot{\varepsilon} & =\Delta u+r, \\
\dot{\varphi} & =\operatorname{div}(u \nabla u)+u r-|\nabla u|^{2},
\end{aligned}
$$

and the requirement (5.2) should be borne in mind. Here $\Delta$ denotes the Laplacian.

5.3. Remarks on the one-phase Stefan problem in $\mathbb{R}$. Consider the simple theory of the last section. Suppose that the underlying space is $\mathbb{R}$, with $\mathscr{B}$ the interval $(0,1)$, and assume that the body is isolated in the sense of the restrictions

$$
q(0, t)=q(1, t)=0
$$

and $r \equiv 0$, with $q(x, t)$, the heat flux, now a scalar field. Suppose that initially there is a single interface, located at $x=s_{0}$, with $\left(0, s_{0}\right)$ solid and $\left(s_{0}, 1\right)$ liquid, and that the initial temperature-deviation $u_{0}(x)$ satisfies

$$
u_{0}(x)=0 \text { for } 0<x<s_{0}
$$

but is otherwise arbitrary. Assume that the resulting interface $x=s(t) \quad\left(s(0)=s_{0}\right)$ evolves smoothly for $t$ small. Then, since $q(0, t)=u(s(t), t)=0$, Eqs. (5.14) reduce to the bulk equations

$$
\begin{aligned}
u(x, t) & =0, & & 0<x<s(t), \\
u_{t} & =u_{x x}, & & s(t)<x<1,
\end{aligned}
$$

in conjunction with the interface conditions

$$
\dot{s}(t)=-u_{x}(s(t), t), \quad u(s(t), t)=0 .
$$

Were the temperature deviation $u$ required to be $\geq 0$ in the liquid, this would be the classical one-phase Stefan problem; since we allow supercooling, there is no such sign restriction on $u$, and the resulting problem is far more difficult (cf. Sherman [15]; Friedman [10]; Fasano and Primicerio [7]; Fasano, Primicerio, and Lacey [9]; Howison, Ockendon, and Lacey [12]; Fasano, Primicerio, Howison, and Ockendon 
[8]). Consider the problem (5.16), (5.18), and (5.19) with $s_{0}=0$, so that $\mathscr{B}$ is initially liquid except for a seed of the solid at $x=0$. If

$$
u_{0}(x)<-1 \text { for } 0<x<1 \text {, }
$$

this problem has no (classical) solution (cf. Fasano, Primicerio, and Lacey [9]); if $u_{0}(x)$ is sufficiently smooth with $u_{0}(0+)=0$ and

$$
\int_{0}^{1} u_{0}(x) d x<-1
$$

then (cf. Sherman [15] and Fasano, Primicerio, and Lacey [9]) there is a solution up to a maximal time $T$, but

$$
\liminf _{t \rightarrow T} \dot{s}^{\cdot}(t)=\infty
$$

I believe that the infinite velocity (5.22) indicates a tendency of the interface to jump at $t=T$, thereby inducing an instantaneous phase change over a spatial interval of nonzero length. I further believe that, while there is no solution with smoothly propagating interface for data consistent with Eq. (5.20), there is a "solution" in which the entire body instantaneously changes phase. In Sec. 6.1 I will discuss formulations that might hopefully account for behavior of this type.

\section{Nucleations.}

6.1. Basic equations. Suppose that, at a fixed time $\lambda$, a portion $\mathscr{A}$ of the liquid is super-supercooled. Then, instantaneously changing the phase of each $x \in \mathscr{A}$ from liquid to solid-holding the internal energy $\varepsilon(x, \lambda)$ fixed-raises the entropy at each $x$ from $\hat{\eta}_{2}(\varepsilon(x, \lambda))$ to $\hat{\eta}_{1}(\varepsilon(x, \lambda))$ or, equivalently, lowers the Gibbs function from $\hat{\varphi}_{2}(\varepsilon(x, \lambda))$ to $\hat{\varphi}_{1}(\varepsilon(x, \lambda))$. Since $\varepsilon(x, \lambda)$ does not change, it is reasonable to expect that such a change is consistent with balance of energy in either the integrated form (2.1) or the distributional form (5.11) . On the other hand, since $\eta(x, t)$ and $\varphi(x, t)$ jump in $t$ at $t=\lambda$ for all $x \in \mathscr{A}$, if volume $(\mathscr{A}) \neq 0$ then such a bulk phase-change, or nucleation, ${ }^{7}$ produces entropy

$$
\int_{\mathscr{A}} \hat{\eta}_{1}(\varepsilon(x, \lambda))-\hat{\eta}_{2}(\varepsilon(x, \lambda)) d V(x)
$$

or, equivalently, dissipates energy

$$
\int_{\mathscr{A}} \hat{\varphi}_{2}(\varepsilon(x, \lambda))-\hat{\varphi}_{1}(\varepsilon(x, \lambda)) d V(x) .
$$

For this reason, application of an entropy or dissipation balance is delicate. In particular, the left side of the dissipation balance (3.3) is not defined at $t=\lambda$, although, presumably, it is $-\infty$, and it is not clear whether or not the distributional dissipation balance $(5.11)_{2}$ has meaning. If the dissipation $\zeta=\nabla u \cdot \mathbf{M} \nabla u$ is integrable on $\mathscr{B} \times(0, T)$, an assumption consistent with Eq. (5.2), then this distributional equation might very well be appropriate. ${ }^{8}$ On the other hand, Eq. $(5.11)_{2}$ might be too strong to include nucleations; in that case we might use Eq. (3.5) distributionally

\footnotetext{
${ }^{7}$ This use of the term includes situations in which an existing interface jumps.

${ }^{8}$ Here I acknowledge valuable discussions with Michiel Bertsch and Roberta Dal Passo.
} 
on $\mathscr{B} \times[0, T)$ and Eq. $(5.11)_{2}$ distributionally on sets $\mathscr{B} \times\left(T_{1}, T_{2}\right)$ that do not include nucleations.

We are therefore led to consider the supercritical Stefan equations even in the presence of nucleations. Nucleations seem physically reasonable; they result in an instantaneous increase in entropy and hence tend to stabilize the system, at least when the actual interfacial entropy is too small to negate the effect of nucleationinducing fluctuations.

Nucleations introduce a seeming lack of uniqueness. For example, if $\mathscr{B}$ is initially liquid with a part $\mathscr{P}$ super-supercooled, and if the body is isolated in the sense of the conditions

$$
\mathbf{q} \cdot \mathbf{n}=0 \quad \text { on } \partial \mathscr{B} \times[0, T), \quad r=0 \quad \text { on } \mathscr{B} \times[0, T),
$$

then I would expect a solution in which portions of $\mathscr{P}$ remain liquid, and another solution in which $\mathscr{P}$ instantaneously changes phase to solid.

6.2. The nucleated Stefan equations. One method of limiting the lack of uniqueness discussed above is to assume that nucleations take place whenever possible. This is equivalent to requiring that super-supercooled liquid instantly undergo an isoenergetic phase change, and similarly for super-superheated solid; mathematically, this is accomplished by adding the constraint

$$
\chi=1 \quad \text { when } \varepsilon<\varepsilon^{*}, \quad \chi=0 \quad \text { when } \varepsilon>\varepsilon^{*} .
$$

We will refer to the distributional equations (5.11) supplemented by the constraint (6.4), the constitutive equations (5.1), and the local equilibrium hypothesis (5.2) as the nucleated Stefan equations.

The constraint (6.4) renders irrelevant the supercritical branches of the constitutive equations. Since the Gibbs functions $\hat{\varphi}_{1}(\varepsilon)$ and $\hat{\varphi}_{2}(\varepsilon)$ coincide at $\varepsilon=\varepsilon^{*}$, this allows us to introduce a combined Gibbs function

$$
\hat{\varphi}(\varepsilon)= \begin{cases}\hat{\varphi}_{1}(\varepsilon) & \text { for } \varepsilon \leq \varepsilon^{*}, \\ \hat{\varphi}_{2}(\varepsilon) & \text { for } \varepsilon \geq \varepsilon^{*}\end{cases}
$$

for the two phases (Fig. 3). We can also define a combined constitutive equation for the temperature deviation by

$$
\hat{u}(\varepsilon)=\hat{\varphi}^{\prime}(\varepsilon)
$$

$\hat{u}(\varepsilon)$ coincides with $\hat{u}_{1}(\varepsilon)$ for $\varepsilon<\varepsilon^{*}$ and with $\hat{u}_{2}(\varepsilon)$ for $\varepsilon>\varepsilon^{*}$ and hence suffers a jump discontinuity at $\varepsilon=\varepsilon^{*}$ with limiting values

$$
u_{1}^{*}:=\hat{u}\left(\varepsilon^{*}-0\right)=\hat{u}_{1}\left(\varepsilon^{*}\right), \quad u_{2}^{*}:=\hat{u}\left(\varepsilon^{*}+0\right)=\hat{u}_{2}\left(\varepsilon^{*}\right) .
$$

The nucleated Stefan equations simplify when the set

$$
\mathscr{A}^{*}(t)=\left\{x: \varepsilon(x, t)=\varepsilon^{*}\right\}
$$

has an empty interior at each $t$, for then the problem reduces to solving the distributional equations (5.11) in conjunction with the local equilibrium hypothesis (5.2) and the constitutive equations (6.5), (6.6), and

$$
\mathbf{q}= \begin{cases}-\mathbf{M}_{1}(u) \nabla u, & \varepsilon<\varepsilon^{*}, \\ -\mathbf{M}_{2}(u) \nabla u, & \varepsilon>\varepsilon^{*}\end{cases}
$$



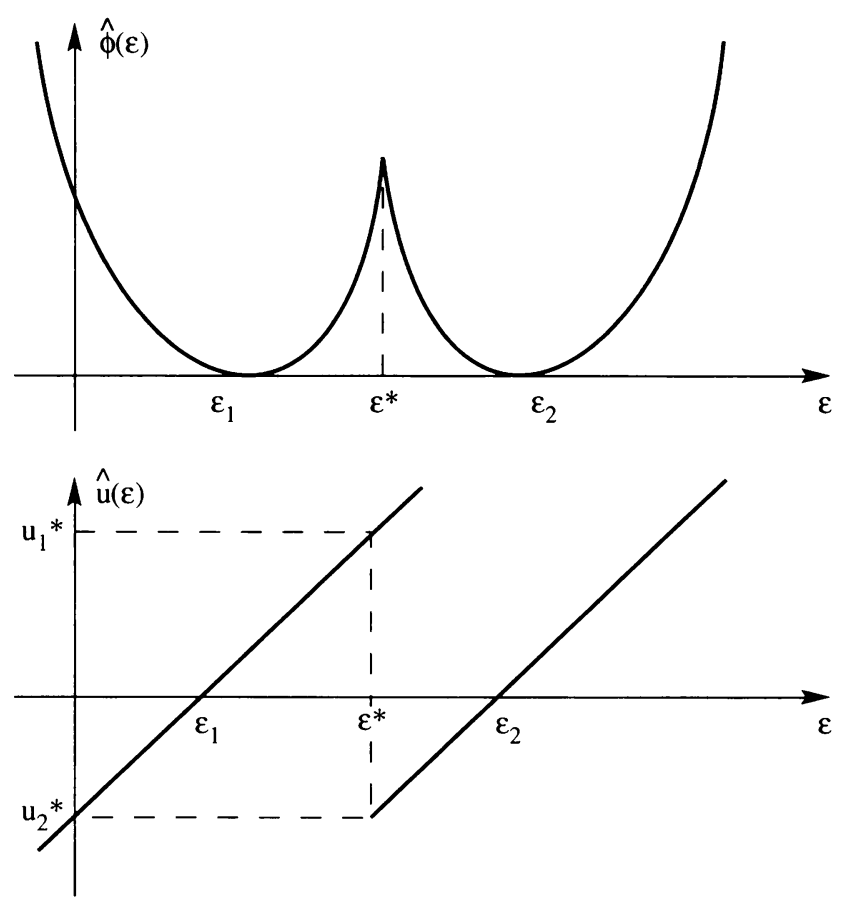

FIG. 3. The Gibbs function $\hat{\varphi}(\varepsilon)$ and temperature deviation $\hat{u}(\varepsilon)$ for the nucleated Stefan equations.

In this formulation the constraint (6.4) as well as all mention of $\chi$ are omitted, and the solid and fluid regions may be defined a posteriori by

$$
\begin{aligned}
& \mathscr{B}_{1}(t)=\left\{x: \varepsilon(x, t)<\varepsilon^{*}\right\}, \\
& \mathscr{B}_{2}(t)=\left\{x: \varepsilon(x, t)>\varepsilon^{*}\right\} .
\end{aligned}
$$

I conjecture that, if the body is isolated, then $\mathscr{A}^{*}(t)$ has an empty interior for all $t>0$.

Note that the initial energy distribution $\varepsilon(x, 0)$ satisfies $\varepsilon(x, 0) \leq \varepsilon^{*}$ in $\mathscr{B}_{1}(0)$ and $\varepsilon(x, 0) \geq \varepsilon^{*}$ in $\mathscr{B}_{2}(0)$. If the solid, say, had just nucleated from a continuous energy distribution, then $\varepsilon(x, 0)$ would be continuous across the interface with value $\varepsilon^{*}$ on the interface; hence the initial temperature deviation would suffer a jump discontinuity at $t=0$ (cf. Eq. (6.7)). If the resulting interface $\mathscr{S}(t)$ evolves smoothly in time, at least for $t>0$ small, then (5.2) and the analysis leading to Eqs. (5.10) tells us that the temperature deviation $u(x, t)$ on $\mathscr{B}(t)$ instantly assumes the value $u(x, t)=0$, and that ${ }^{9}$ for all sufficiently small $t$ the equations reduce to

$$
\begin{aligned}
\dot{\varepsilon} & =-\operatorname{div} \mathbf{q}+r, & & \varepsilon \leq \varepsilon^{*}, \\
u & =\hat{u}_{1}(\varepsilon), & & \mathbf{q}=-\mathbf{M}_{1}(u) \nabla u
\end{aligned}
$$

in the solid;

$$
\begin{aligned}
\dot{\varepsilon} & =-\operatorname{div} \mathbf{q}+r, & & \varepsilon \geq \varepsilon^{*}, \\
u & =\hat{u}_{2}(\varepsilon), & & \mathbf{q}=-\mathbf{M}_{2}(u) \nabla u
\end{aligned}
$$

\footnotetext{
${ }^{9}$ I expect no new nucleations if the system is isolated.
} 
in the liquid; and

$$
l V=[\mathbf{q}] \cdot \mathbf{m}, \quad u=0
$$

on the interface.

6.3. Model equations for behavior near the transition temperature. For the model equations $(4.13)-(4.18)$, the nucleated Stefan equations $(6.11)-(6.13)$ reduce to ${ }^{10}$

$$
\begin{array}{lll}
\dot{u}=\Delta u+r, & u \leq \frac{1}{2} & \text { in the solid, } \\
\dot{u}=\Delta u+r, & u \geq-\frac{1}{2} & \text { in the liquid, } \\
V=-[\nabla u] \cdot \mathbf{m}, & u=0 & \text { on the interface }
\end{array}
$$

for the temperature deviation, or

$$
\begin{aligned}
\dot{\varepsilon} & =\Delta \varepsilon+r, & \varepsilon & \leq \frac{1}{2} & & \text { in the solid, } \\
\dot{\varepsilon} & =\Delta \varepsilon+r, & \varepsilon & \geq \frac{1}{2} & & \text { in the liquid, } \\
V & =-[\nabla u] \cdot \mathbf{m}, & \varepsilon_{S} & =0, \quad \varepsilon_{L}=1 & & \text { on the interface }
\end{aligned}
$$

for the internal energy, where $\varepsilon_{S}$ and $\varepsilon_{L}$ denote the limiting values of the internal energy from the solid and liquid, respectively.

REMARK 6.1. Within this framework-in which nucleations take place whenever possible-we can allow for different specific heats in the solid and liquid without introducing erroneous behavior: since we require that the material be solid when $\varepsilon<\varepsilon^{*}$ and liquid when $\varepsilon>\varepsilon^{*}$, the spurious change in stability for $\varepsilon=\varepsilon^{* *}$ (cf. Remark 4.1) cannot manifest itself. In this case, for $c_{1}=c, c_{2}=1, \varepsilon_{1}=0, \varepsilon_{2}=1$,

$$
\varepsilon^{*}=\sqrt{c} /(1+\sqrt{c}), \quad u_{1}^{*}=c^{-1} \varepsilon^{*}, \quad u_{2}^{*}=\varepsilon^{*}-1
$$

(cf. Eqs. (6.7)). In this case, if we retain Eq. (4.18) as the constitutive equation for the heat flux, we are led to the following generalization of Eqs. (6.14):

$$
\begin{aligned}
& c u^{i}=\Delta u+r, \quad u \leq c^{-1} \varepsilon^{*} \quad \text { in the solid, } \\
& \dot{u}=\Delta u+r, \quad u \geq \varepsilon^{*}-1 \quad \text { in the liquid, } \\
& V=-[\nabla u] \cdot \mathbf{m}, \quad u=0 \quad \text { on the interface; }
\end{aligned}
$$

with obvious modifications we can also account for different conductivities in the two phases.

6.4. Further discussion of the one-phase problem in $\mathbb{R}$. We now return to the one-phase Stefan problem based on the model equations (4.13)-(4.18). Consider first initial data consistent with Eq. (5.20), in which case the problem as presented in Sec. 5.3 has no solution. Within the framework of Sec. 6.1 (nucleations allowed but not required), this problem has a (probably nonunique) solution in which the entire

\footnotetext{
${ }^{10}$ Visintin [18] proposes a problem of this type to model nucleations, but his bounds $u \leq a_{1}$ in the solid and $u \geq-a_{2}$ in the liquid $\left(a_{1}, a_{2}>0\right)$ are independent constitutive quantities unrelated to the Gibbs functions $\hat{\varphi}_{1}(\varepsilon)$ and $\hat{\varphi}_{2}(\varepsilon)$. I believe that this could possibly lead to inconsistencies.
} 
interval $(0,1)$ changes phase to solid, the change being isoenergetic and instantaneous. If we consider the nucleated Stefan equations (nucleations occur whenever possible), then, by Eqs. (6.14), the temperature deviation in the liquid must satisfy the constraint $u(x, t) \geq-\frac{1}{2}=u_{2}^{*}$. Thus Eq. $(5.20)$, with $(0,1)$ liquid, is inadmissible initial data. (Such data is rendered admissible by an isoenergetic phase change of the entire interval to solid.) If we allow the specific heats in the two phases to differ, as in Eqs. (6.17), then, by Eqs. (6.16), the lowest possible value for the limiting temperature-derivation $u_{2}^{*}$ in the liquid is -1 (for $c=0$ ), so that Eq. (5.20) remains inadmissible.

Consider next initial data consistent with Eq. (5.21). For the nucleated Stefan equations such data, with $(0,1)$ liquid, is inadmissible; it is rendered admissible by an isoenergetic phase change, to solid, of points $x$ with $u_{0}(x)<\varepsilon^{*}-1$. On the other hand, within the framework of Sec. 6.1, the data (5.21) is admissible. According to Fasano, Primicerio, Howison, and Ockendon [8], the singularity (5.22) at $t=T$ occurs when the interface contacts the $u=-1$ level set. In this case, if the set $\{x: u(x, T-0) \leq-1\}$ has a connected component of the form $[s(T-0), Y]$, I would expect that the solution can be continued past $t=T$ using an isoenergetic phase change of $[s(T-0), Y]$ to solid at time $T$, and then starting the interface smoothly again from $Y$ at $T$ (see Fig. 4 on p. 152). Working within the framework of the simple model (4.13)-(4.18) (with equal specific heats) the temperature distribution in the solid $(0, Y)$ at time $T+0$ is generally no longer $u=0:$ it is determined by setting the energy of the solid material in $[s(T-0), Y]$ equal to the energy in $[s(T-0), Y]$ in the liquid phase at $t=T-0$; by Eq. $(5.13)_{2}$,

$$
u(x, T+0)=1+u(x, T-0), \quad s(T) \leq x \leq Y .
$$

The resulting problem, now two-phase, consists in solving

$$
\begin{aligned}
& u_{t}=u_{x x}, \quad x \neq s(t) ; \\
& s^{\cdot}(t)=u_{x}(s(t)-0, t)-u_{x}(s(t)+0, t) ; \\
& u(s(t), t)=0 ; \\
& u(x, T+0)=g(x), \quad 0<x<L ; \\
& s(T+0)=Y ; \quad u_{x}(0, t)=u_{x}(L, t)=0
\end{aligned}
$$

for $t>T$, where $g(x)$ is zero on $(0, s(T))$, is given by Eq. (6.18) on $[s(T), Y]$, and is given by $u(x, T-0)$ on $(Y, L)$.

If we neglect the conductivity of the solid, then the problem for $t>T$ is the one-phase problem: ${ }^{11}$

\footnotetext{
${ }^{11}$ For solid-liquid systems the individual conductivities are generally equal in order of magnitude, as are the specific heats, so a two-phase theory seems more suitable. A one-phase theory is often applied to mass transport (cf. Remark 3.3), where the diffusivity within the solid is usually orders of magnitude lower than that within the liquid.

I believe that Eqs. (6.20) are valid for $\dot{s}^{\circ}(t) \geq 0$, but for $\dot{s}^{\circ}(t)<0$ there are problems when the interface impinges on solid material with nonzero temperature deviation. The correct formulation (within a continuum thermodynamical framework) of the one-phase problem seems to me an open issue.
} 


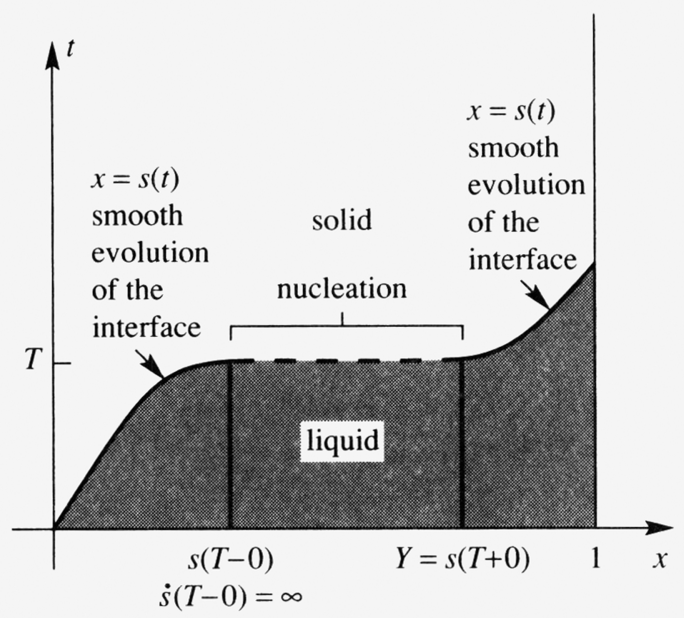

Fig. 4. Interfacial motion in which the interface jumps at time $T$ indicating a nucleation.

$$
\begin{aligned}
& u_{t}=u_{x x}, \quad s(t)<x<L ; \\
& \dot{s}(t)=-u_{x}(s(t), t) ; \quad u(s(t), t)=0 ; \\
& u(x, T+0)=u(x, T-0), \quad Y<x<L ; \\
& s(T+0)=Y ; \quad u_{x}(L, t)=0 .
\end{aligned}
$$

There is an apparent lack of uniqueness in the continuation process described above for both Eqs. (6.19) and (6.20). I believe that a solution can also be continued using as nucleation interval any super-supercooled interval $[s(T-0), Z]$ with $Z \geq Y$ and $u(Z, T-0) \geq-1$. A possible method of investigating this issue is to allow for kinetic undercooling, ${ }^{12}$ which leads to the regularized problem:

$$
\begin{aligned}
& u_{t}=u_{x x}, \quad s(t)<x<L ; \\
& \dot{s}(t)=-u_{x}(s(t), t) ; \quad u(s(t), t)=-b s^{\circ}(t) ; \\
& u(x, 0)=u_{0}(x), \quad 0<x<L ; \\
& s(0)=0 ; \quad u_{x}(L, t)=0,
\end{aligned}
$$

with $b>0$. Given initial data $u_{0}(x)$ consistent with Eq. (5.21), an interesting question is: Does the limit as $b \rightarrow 0$ of the solution of Eq. (6.21) exist, and if so is it a solution of one of the continuation processes described above? I would expect the limit to be the nucleation to $Y$. The nucleations to $Z>Y$ have $u(Z, T-0)>-1$ with $Z$ super-supercooled at $t=T-0$ for the model with equal specific heats. But the one-phase problem (6.20) makes no mention of the specific heat of the solid, and, for $u(Z, T-0)>-1$ there is always a choice of specific heat for the solid such that $Z$ is not super-supercooled at $T-0$ (cf. Eqs. (6.16)).

\footnotetext{
${ }^{12}$ Cf., e.g., Visintin [17], Gurtin [11], Dewynne, Howison, Ockendon, and Xie [6]; the latter authors refer to unpublished work of Visintin and Xie for the existence, uniqueness, and regularity of solutions.
} 
A simpler framework within which to study this issue is to consider Eqs. (6.21) with and without $b=0$ for $s(0)=0$ and initial data $u_{0}(x)$ that is smooth on $[0,1]$ and of the form

$$
\begin{array}{cc}
u_{0}(0)=u_{0}(Y)=-1 ; \\
u_{0}(x)<-1, & 0<x<Y ; \\
u_{0}(x)>-1, & Y<x \leq L .
\end{array}
$$

Note added in proof. I have recently seen a preprint, "Two-phase Stefan problem with supercooling", by I. G. Götz and B. Zaltzman, who study the two-phase problem with kinetic undercooling and show that as the kinetic modulus $b \rightarrow 0$, the limiting solution contains a jump from $s(T-0)$ to $Y$, with $Y$ as in the paragraph containing (6.18). It would be interesting to see whether their limiting solution obeys the nucleation condition (6.18).

7. Fine phase mixtures. Stabilized solutions. Mushy zones. Consider the Gibbs function $\hat{\varphi}(u)$ corresponding to the nucleated Stefan equations (cf. Eq. (6.5)). The convexification $\varphi_{f}(\varepsilon)$ of this function coincides with $\hat{\varphi}(\varepsilon)$ for $\varepsilon \leq \varepsilon_{1}$ and $\varepsilon \geq \varepsilon_{2}$, while for $\varepsilon_{1} \leq \varepsilon \leq \varepsilon_{2}$ the graph of $\varphi_{f}(\varepsilon)$ is the line between $\left(\varepsilon_{1}, 0\right)$ and $\left(\varepsilon_{2}, 0\right)$. Let

$$
u_{f}(\varepsilon):=\varphi_{f}^{\prime}(\varepsilon),
$$

so that $u=u_{f}(\varepsilon)$ is the temperature deviation in a material whose Gibbs function is given by $\varphi=\varphi_{f}(\varepsilon)$.

Material whose energy $\varepsilon$ lies between $\varepsilon_{1}$ and $\varepsilon_{2}$ is unstable to perturbations at constant energy. Indeed, the Gibbs function is lowered by letting the material develop a fine phase mixture, of the same energy $\varepsilon$, consisting of volume fractions $\nu_{1}$ and $\nu_{2}$ of solid at energy $\varepsilon_{1}$ and liquid at energy $\varepsilon_{2}$ :

$$
\begin{aligned}
& \varepsilon=\nu_{1} \varepsilon_{1}+\nu_{2} \varepsilon_{2}, \quad \nu_{1}+\nu_{2}=1, \\
& \nu_{1} \hat{\varphi}\left(\varepsilon_{1}\right)+\nu_{2} \hat{\varphi}\left(\varepsilon_{2}\right)=\varphi_{f}(\varepsilon)<\hat{\varphi}(\varepsilon) .
\end{aligned}
$$

Consider now a solution of the nucleated Stefan equations. Let $t$ denote a fixed time, and let

$$
\mathscr{U}(t)=\left\{x: \varepsilon(x, t) \in\left[\varepsilon_{1}, \varepsilon_{2}\right]\right\} .
$$

Then the instantaneous formation, at each $x \in \mathscr{U}(t)$, of a fine phase mixture of $\varepsilon_{1}$ and $\varepsilon_{2}$,

$$
\varepsilon(x, t)=\nu_{1}(x, t) \varepsilon_{1}+\nu_{2}(x, t) \varepsilon_{2}, \quad \nu_{1}(x, t)+\nu_{2}(x, t)=1,
$$

lowers the integral of $\varphi(x, t)$ over $\mathscr{U}(t)$ by the amount

$$
\int_{\mathscr{L}(t)}\left(\varphi_{f}(\varepsilon(x, t))-\hat{\varphi}(\varepsilon(x, t))\right) d V(x)
$$


but keeps the energy of any subset of $\mathscr{U}(t)$ unchanged. Such fine phase mixtures stabilize the solution, and, arguing as in Sec. 6, it seems physically reasonable to consider solutions for which these instantaneous changes always take place. For such solutions we replace the Gibbs function $\hat{\varphi}(\varepsilon)$ by its convexification $\varphi_{f}(\varepsilon)$, and, if we consider fine phase mixtures as limits of regions of constant energies $\varepsilon_{1}$ or $\varepsilon_{2}$, then the limiting value of the corresponding temperature deviation is $\hat{u}\left(\varepsilon_{1}\right)=\hat{u}\left(\varepsilon_{2}\right)=$ $u_{f}(\varepsilon)=0$. Thus allowing for the instantaneous formation of fine phase mixtures should be equivalent to replacing $\hat{\varphi}(\varepsilon)$ and $\hat{u}(\varepsilon)$ by $\varphi_{f}(\varepsilon)$ and $u_{f}(\varepsilon)$, respectively, and hence considering the constitutive equations

$$
\varphi=\varphi_{f}(\varepsilon), \quad u=u_{f}(\varepsilon), \quad q=-\mathbf{M}(u) \nabla u,
$$

with $\mathbf{M}(u)=\mathbf{M}_{1}(u)$ for $u \leq 0$ and $\mathbf{M}(u)=\mathbf{M}_{2}(u)$ for $u>0$.

Consider a distributional solution $\varepsilon(x, t)$ on $\mathscr{B} \times(0, T)$ of the energy equation (2.3) with $u(x, t)$ subject to the local equilibrium hypothesis (5.2). Because of the strict monotonicity of the function $u_{f}$ outside $\left[\varepsilon_{1}, \varepsilon_{2}\right]$, spatial discontinuities in $\varepsilon$ can occur only at energies within $\left[\varepsilon_{1}, \varepsilon_{2}\right]$, and hence only at $u=0$, and so we need not consider the dissipation balance. We will refer to the distributional energy equation (2.3) - supplemented by (5.2) and (7.6) - as the stabilized Stefan equations.

The set $\mathscr{U}(t)$ defined in Eq. (7.3) is also the set of $x$ such that $u(x, t)=0$. The set $\mathscr{M}(t):=\operatorname{Int} \mathscr{U}(t)$ represents a mushy zone ${ }^{13}$ containing a fine phase mixture of liquid and solid. Assume that $r \equiv 0$. Fix the time $t$ and suppress it in what follows. Since $\nabla u=0$ in $\mathscr{M}$, Eqs. (7.6) yield $\mathbf{q}=\mathbf{0}$ for $x \in \mathscr{M}$; thus $\varepsilon=0$ in $\mathscr{M}$. Consider a point $y$ near which $\mathscr{M}$ and the solid region $\mathscr{B}_{1}$ are separated by a surface $\mathscr{J}$ containing $y$. Then, since $\varepsilon \leq \varepsilon_{1}$ in $\mathscr{B}_{1}$ and $\varepsilon \geq \varepsilon_{1}$ in $\mathscr{M},[\varepsilon] \geq 0$ at $y$, where [] here denotes the limiting value in the mush minus that in the solid. Assume, further, that the solid is isotropic, so that $\mathbf{q}$ is parallel to (and points in the opposite direction of) $\nabla u$ in $\mathscr{B}_{1}$. Let $\mathbf{m}$ denote the normal to $\mathscr{J}$ directed outward from the solid, and let $V$ denote the corresponding normal velocity. Then, since $u \leq 0$ in the solid, [q] $\mathbf{m} \leq 0$ at $y$, and, by the analog of Eq. (5.4),$V \leq 0$, so that the mushy region cannot grow into the solid. Similarly, the mushy region cannot grow into the liquid. What we have formally shown is that, for sufficiently regular solutions, the mushy region decreases monotonically; in fact, $\mathscr{M}(t)$ nests as $t$ increases in the sense that $\mathscr{M}(t) \subset \mathscr{M}(\lambda)$ for $t>\lambda$. I conjecture that this is a generic property of weak solutions of the stabilized Stefan equations with $r \equiv 0$. I conjecture further that if the body is isolated (cf. Eqs. (6.3)) and initially not all mush, then the volume of the mushy region tends to zero in finite time. ${ }^{14}$

Finally, we remark that in the absence of a mushy zone the basic equations are those of the classical Stefan problem.

Acknowledgment. I greatly acknowledge valuable discussions with Fred Almgren, Peter Bates, Michiel Bertsch, Roberta Dal Passo, Paul Fife, Giorgios Kosioris,

\footnotetext{
${ }^{13}$ Such mushy zones are known to occur in solutions of the Stefan problem with nonzero heat supply (cf., e.g., Lacey and Taylor [13], Ughi [16], Crowley and Ockendon [4]). Detailed studies of the qualitative properties of the mushy zone are given by Bertsch, De Mottoni, and Peletier [1, 2].

${ }^{14}$ A result of this type, for $\mathscr{B}=[0,1]$, is given by Bertsch, De Mottoni, and Peletier [1].
} 
Stephan Luckhaus, Mete Soner, and Augusto Visintin. This work was supported by the Army Research Office and by the National Science Foundation.

\section{REFERENCES}

[1] M. Bertsch, P. De. Mottoni, and L. A. Peletier, Degenerate diffusion and the Stefan problem, Nonlin. Anal. 8, 1311-1336 (1984)

[2] M. Bertsch, P. De. Mottoni, and L. A. Peletier, The Stefan problem with heating. Appearance and disappearance of a mushy region, Trans. Amer. Math. Soc. 293, 677-691 (1986)

[3] B. D. Coleman and W. Noll, The thermodynamics of elastic materials with heat conduction and viscosity, Arch. Rational Mech. Anal. 13, 245-261 (1963)

[4] A. B. Crowley and J. R. Ockendon, Modelling mushy regions, Appl. Sci. Res. 44, 1-7 (1987)

[5] C. M. Dafermos, Hyperbolic systems of balance laws, Systems of Nonlinear Partial Differential Equations, J. M. Ball ed., Reidel, Dordrecht, 1983

[6] J. N. Dewynne, S. D. Howison, J. R. Ockendon, and W. Xie, Asymptotic behavior of solutions to the Stefan problem with a kinetic condition at the free boundary, J. Austral. Math. Soc. Ser. B 31, 81-96 (1989)

[7] A. Fasano and M. Primicerio, General free boundary problems for the heat equation, Parts 1-3, J. Math. Anal. Appl. 57, 694-723; 58, 202-231; 59, 1-14 (1977)

[8] A. Fasano, M. Primicerio, S. D. Howison, and J. R. Ockendon, Some remarks on the regularization of supercooled one-phase Stefan problems in one dimension, Quart. Appl. Math. 48, 153-168 (1990)

[9] A. Fasano, M. Primicerio, and A. A. Lacey, New results on some classical parabolic free boundary problems, Quart. Appl. Math. 38, 439-460 (1981)

[10] A. Friedman, Analyticity of the free boundary for the Stefan problem, Arch. Rational Mech. Anal. 61, 97-125 (1976)

[11] M. E. Gurtin, Multiphase thermomechanics with interfacial structure. 1. Heat conduction and the capillarity balance law, Arch. Rational. Mech. Anal. 104, 195-221 (1988)

[12] S. D. Howison, J. R. Ockendon, and A. A. Lacey, Singularity development in moving boundary problems, Quart. Appl. Math. 38, 343-360 (1985)

[13] A. A. Lacey and A. B. Taylor, A mushy region in a Stefan problem, IMA J. Appl. Math. 30, 303-314 (1983)

[14] S. Luckhaus, Solutions for the two-phase Stefan problem with the Gibbs-Thomson law for the melting temperature, Euro. J. Appl. Math. 1, 101-111 (1990)

[15] B. Sherman, A general one-phase Stefan problem, Quart. Appl. Math. 28, 377-382 (1970)

[16] M. Ughi, A melting problem with a mushy region, IMA J. Appl. Math. 33, 135-152 (1984)

[17] A. Visintin, A new model for supercooling and superheating effects, IMA J. Appl. Math. 36, 141-157 (1984)

[18] A. Visintin, Stefan problem with surface tension, Mathematical Models for Phase Change Problems, J. F. Rodrigues ed., Birkhäuser Verlag, New York, 1989

[19] A. Visintin, Surface tension effects in phase transition, Material Instabilities in Continuum Mechanics, J. M. Ball ed., Clarendon Press, New York, 1988 